\title{
Fibonacci Lacunary Ideal Convergence of Double Sequences in Intuitionistic Fuzzy Normed Linear Spaces
}

\author{
Ömer Kişi
}

\begin{abstract}
The purpose of this article is to research the concept of Fibonacci lacunary ideal convergence of double sequences in intuitionistic fuzzy normed linear spaces (IFNS). Additionally, a new concept, called Fibonacci lacunary convergence, is examined. Fibonacci lacunary $\mathcal{I}_{2}$-limit points and Fibonacci lacunary $\mathcal{I}_{2}$-cluster points for double sequences in IFNS have been defined and the significant results have been given. Additionally, Fibonacci lacunary Cauchy and Fibonacci lacunary $\mathcal{I}_{2}$-Cauchy double sequences in IFNS are worked.
\end{abstract}

Keywords: Fibonacci sequence; intuitionistic fuzzy normed linear space; limit point; cluster point.

AMS Subject Classification (2020): Primary: 40A30; Secondary: $40 G 15$.

\section{Introduction and Background}

Statistical convergence of single and double real sequences was firstly studied by Fast [5] and Mursaaleen and Edely [17], respectively.

$\mathcal{I}$-convergence idea was firstly considered by Kostyrko et al. [16]. Tripathy et al. [29] gave the concept of ideal convergence of double sequences in a metric space and examined fundamental features.

Using lacunary sequence, Fridy and Orhan [6] examined lacunary statistical convergence. Lacunary statistical convergence of double sequences was worked at initial stage by Savaş and Patterson [24]. Lacunary ideal convergence of real sequences was introduced by Tripathy et al. [28]. This kind of convergence extended from single to double sequences with the study of Hazarika [8]. For different studies on these topic we refer to [3, 4, 21].

After the original study of Zadeh [30], a huge number of research works have appeared on fuzzy theory and its applications as well as fuzzy analogues of the classical theories. Fuzzy sets (FS), have been extensively applied in different disciplines and technologies. The theory of intuitionistic fuzzy sets (IFS) was presented by Atanassov [1]. The FS and IFS have been extensively to analyse many complex problems associated with different fields, particularly in decision-making. In IFS, membership degrees are described with a pair of a membership degree and 
a nonmembership degree. Intuitionistic fuzzy metric space was investigated by Park [22]. In [23], motivated by Park's definition of an IF-metric, Lael and Nourouzi first defined an IF-normed space. Statistical convergence of single and double sequences in IFNS was defined by Karakuş et al [11] and Mursaleen et al [18], respectively. Some researches of convergence of sequences in some normed linear spaces in a fuzzy settings can be found in $[2,19,20]$. Also, similar works worked by some authors, see [26, 27].

Fibonacci gave Fibonacci sequences which was published in the book 'Liber Abaci'. This sequences were earlier stated as Virahanka numbers by Indian mathematics [7]. The sequence

$$
(1,1,2,3,5,8,13,21,34,55,89,144, \ldots)
$$

is known as Fibonacci sequence [15].

Kara and Başarır [9] prensented the first applications of Fibonacci sequence in the sequence spaces. Then, Kara [10] acquired the Fibonacci difference matrix $\widehat{F}$ via Fibonacci sequence $\left(f_{n}\right)$ and described some new sequence spaces in this connection. Recently, Kirişci [12] thought the Fibonacci statistical convergence on IFNS. Kişi and Tuzcuoğlu [13] examined Fibonacci lacunary statistical convergence on IFNS. Additionally, Fibonacci ideal convergence of double sequences in IFNS was worked by Kişi and Güler [14].

Let us start with fundamental definitions from the literature.

Let $\emptyset \neq S$ be a set, and then $\emptyset \neq \mathcal{I} \subseteq P(S)$ is said to be an ideal on $S$ iff $(i) \emptyset \in \mathcal{I}$, (ii) $\mathcal{I}$ is additive under union, (iii) for each $A \in \mathcal{I}$ and each $B \subseteq A$ we get $B \in \mathcal{I}$. A non-empty family of sets $\mathcal{F}$ is called filter on $S$ iff $(i) \emptyset \notin \mathcal{F}$, (ii) for each $A, B \in \mathcal{F}$ we get $A \cap B \in \mathcal{F}$, (iii) for every $A \in \mathcal{F}$ and each $B \supseteq A$, we obtain $B \in \mathcal{F}$. Correlation between ideal and filter is specified as follows:

$$
\mathcal{F}(\mathcal{I})=\left\{K \subset S: K^{c} \in \mathcal{I}\right\},
$$

where $K^{c}=S-K$.

A non-trivial ideal $\mathcal{I}_{2}$ of $\mathbb{N} \times \mathbb{N}$ is named as strongly admissible if $\{i\} \times \mathbb{N}$ and $\mathbb{N} \times\{i\}$ belong to $\mathcal{I}_{2}$ for each $i \in \mathbb{N}$.

Throughout the paper, we take $\mathcal{I}_{2}$ as a strongly admissible ideal in $\mathbb{N} \times \mathbb{N}$.

Let $(X, \rho)$ be a metric space. A double sequence $x=\left(x_{m n}\right)$ is named as $\mathcal{I}_{2}$-convergent to $\xi$, if for any $\varepsilon>0$ we get $P(\varepsilon)=\left\{(m, n) \in \mathbb{N} \times \mathbb{N}: \rho\left(x_{m n}, \xi\right) \geq \varepsilon\right\} \in \mathcal{I}_{2}$. In this case, we write

$$
\mathcal{I}_{2}-\lim _{m, n \rightarrow \infty} x_{m n}=\xi
$$

A double sequence $\bar{\theta}=\theta_{u s}=\left\{\left(k_{u}, l_{s}\right)\right\}$ is named as double lacunary sequence if there are two increasing sequences of integers $\left(k_{u}\right)$ and $\left(l_{s}\right)$ such that

$$
k_{0}=0, h_{u}=k_{u}-k_{u-1} \rightarrow \infty \text { and } l_{0}=0, \bar{h}_{s}=l_{s}-l_{s-1} \rightarrow \infty, u, s \rightarrow \infty .
$$

We utilize the subsequent notations

$$
k_{u s}:=k_{u} l_{s}, h_{u s}:=h_{u} \bar{h}_{s}
$$

and $\theta_{u s}$ is determined by

$$
\begin{gathered}
J_{u s}:=\left\{(k, l): k_{u-1}<k \leq k_{u} \text { and } l_{s-1}<l \leq l_{s}\right\}, \\
q_{u}:=\frac{k_{u}}{k_{u-1}}, \bar{q}_{s}:=\frac{l_{s}}{l_{s-1}} \text { and } q_{u s}:=q_{u} \bar{q}_{s} .
\end{gathered}
$$

Throughout the paper, by $\theta_{2}=\theta_{u s}=\left\{\left(k_{u}, l_{s}\right)\right\}$ we will indicate a double lacunary sequence.

Schweizer and Sklar [25] defined continuous t-norm and t-conorm. Using the continuous t-norm and t-conorm, Lael and Nourouzi [23] defined the concept of IFNS as follows:

The five-tuple $(X, \phi, \omega, *, \diamond)$ is named as IFNS if $X$ is a vector space, $*$ is a continuous t-norm, $\diamond$ is a continuous t-conorm and $\phi, \omega$ are fuzzy sets on $X \times(0, \infty)$ fulfilling the subsequent conditions: For every $a, b \in X$ and $p, q>0$ :

(i) $\phi(a, q)+\omega(a, q) \leq 1$,

(ii) $\phi(a, q)>0$,

(iii) $\phi(a, q)=1$ if and only if $a=0$,

(iv) $\phi(c a, q)=\phi\left(a, \frac{q}{|c|}\right)$ if $c \neq 0$,

(v) $\phi(a, q) * \phi(b, p) \leq \phi(a+b, q+p)$,

(vi) $\phi(a,):.(0, \infty) \rightarrow[0,1]$ is continuous in $q$;

(vii) $\lim _{q \rightarrow \infty} \phi(a, q)=1$ and $\lim _{q \rightarrow 0} \phi(a, q)=0$, 
(viii) $\omega(a, q)<1$,

$(\imath x) \omega(a, q)=0$ if and only if $a=0$,

$(x) \omega(c a, q)=\omega\left(a, \frac{q}{|c|}\right)$ if $c \neq 0$,

$(x \imath) \omega(a, q) \diamond \omega(b, p) \geq \omega(a+b, q+p)$,

$(x \imath) \omega(a,):.(0, \infty) \rightarrow[0,1]$ is continuous in $q$;

(xurı) $\lim _{q \rightarrow \infty} \omega(a, q)=0$ and $\lim _{q \rightarrow 0} \omega(a, q)=1$.

\section{Main Results}

Definition 2.1. Let $(X, \phi, \omega, *, \diamond)$ be an IFNS. A double sequence $x=\left(x_{k l}\right)$ in $X$ is named as Fibonacci lacunary convergent to $\xi$ with regards to the $\operatorname{IFN}(\phi, \omega)$ if, for every $t>0$ and $\varepsilon \in(0,1)$, there is $r_{0} \in \mathbb{N}$ such that

$$
\frac{1}{h_{u s}} \sum_{(k, l) \in J_{u s}} \phi\left(\widehat{F} x_{k l}-\xi, t\right)>1-\varepsilon \text { and } \frac{1}{h_{u s}} \sum_{(k, l) \in J_{u s}} \omega\left(\widehat{F} x_{k l}-\xi, t\right)<\varepsilon
$$

for all $u, s \geq r_{0}$. In this case, we write $(\phi, \omega)^{\theta_{u s}}-\lim F x=\xi$.

Theorem 2.1. If $(\phi, \omega)^{\theta_{u s}}-\lim F x=\xi$, then $(\phi, \omega)^{\theta_{u s}}-\lim F x$ is unique.

Proof. Presume that $(\phi, \omega)^{\theta_{u s}}-\lim F x=\xi_{1}$ and $(\phi, \omega)^{\theta_{u s}}-\lim F x=\xi_{2}$. Given $\varepsilon>0$, select $\gamma \in(0,1)$ such that $(1-\gamma) *(1-\gamma)>1-\varepsilon$ and $\gamma \diamond \gamma<\varepsilon$. Now, for all $t>0$, there is $r_{1} \in \mathbb{N}$ such that

$$
\frac{1}{h_{u s}} \sum_{(k, l) \in J_{u s}} \phi\left(\widehat{F} x_{k l}-\xi_{1}, t\right)>1-\varepsilon \text { and } \frac{1}{h_{u s}} \sum_{(k, l) \in J_{u s}} \omega\left(\widehat{F} x_{k l}-\xi_{1}, t\right)<\varepsilon
$$

for all $u, s \geq r_{1}$. Also, there is $r_{2} \in \mathbb{N}$ such that

$$
\frac{1}{h_{u s}} \sum_{(k, l) \in J_{u s}} \phi\left(\widehat{F} x_{k l}-\xi_{2}, t\right)>1-\varepsilon \text { and } \frac{1}{h_{u s}} \sum_{(k, l) \in J_{u s}} \omega\left(\widehat{F} x_{k l}-\xi_{2}, t\right)<\varepsilon
$$

for all $u, s \geq r_{2}$. Consider $r_{0}=\max \left\{r_{1}, r_{2}\right\}$. Then, for $u, s \geq r_{0}$, we take a $(m, p) \in \mathbb{N} \times \mathbb{N}$ such that

$$
\phi\left(\widehat{F} x_{m p}-\xi_{1}, \frac{t}{2}\right)>\frac{1}{h_{u s}} \sum_{(k, l) \in J_{u s}} \phi\left(\widehat{F} x_{k l}-\xi_{1}, \frac{t}{2}\right)>1-\gamma
$$

and

$$
\phi\left(\widehat{F} x_{m p}-\xi_{2}, \frac{t}{2}\right)>\frac{1}{h_{u s}} \sum_{(k, l) \in J_{u s}} \phi\left(\widehat{F} x_{k l}-\xi_{2}, \frac{t}{2}\right)>1-\gamma .
$$

Then, we obtain

$$
\begin{aligned}
\phi\left(\xi_{1}-\xi_{2}, t\right) & \geq \phi\left(\widehat{F} x_{m p}-\xi_{1}, \frac{t}{2}\right) * \phi\left(\widehat{F} x_{m p}-\xi_{2}, \frac{t}{2}\right) \\
& >(1-\gamma) *(1-\gamma)>1-\varepsilon .
\end{aligned}
$$

Since $\varepsilon>0$ is abritrary, we have $\phi\left(\xi_{1}-\xi_{2}, t\right)=1$ for every $t>0$, which gives that $\xi_{1}=\xi_{2}$.

Definition 2.2. A double sequence $x=\left(x_{k l}\right)$ in IFNS is named as Fibonacci lacunary $\mathcal{I}_{2}$-convergent to $\xi$ with regards to the $\operatorname{IFN}(\phi, \omega)$ if, for each $\varepsilon>0$ and $t>0$, the set

$$
\left\{\begin{array}{c}
(u, s) \in \mathbb{N} \times \mathbb{N}: \frac{1}{h_{u s}} \sum_{(k, l) \in J_{u s}} \phi\left(\widehat{F} x_{k l}-\xi, t\right) \leq 1-\varepsilon \\
\text { or } \frac{1}{h_{u s}} \sum_{(k, l) \in J_{u s}} \omega\left(\widehat{F} x_{k l}-\xi, t\right) \geq \varepsilon
\end{array}\right\} \in \mathcal{I}_{2} .
$$

$\xi$ is named the Fibonacci $\mathcal{I}_{\theta}$-limit of the sequence of $\left(x_{k l}\right)$, and we note $F \mathcal{I}_{\theta_{u s}}^{(\phi, \omega)}-\lim x=\xi$. 
Lemma 2.1. For every $\varepsilon>0$ and $t>0$, the following demonstrations are equivalent.

(a) $F \mathcal{I}_{\theta_{u s}}^{(\phi, \omega)}-\lim x=\xi$,

(b) $\left\{(u, s) \in \mathbb{N} \times \mathbb{N}: \frac{1}{h_{u s}} \sum_{(k, l) \in J_{u s}} \phi\left(x_{k l}-\xi, t\right) \leq 1-\varepsilon\right\} \in \mathcal{I}_{2}$ and

$\left\{(u, s) \in \mathbb{N} \times \mathbb{N}: \frac{1}{h_{u s}} \sum_{(k, l) \in J_{u s}} \omega\left(x_{k l}-\xi, t\right) \geq \varepsilon\right\} \in \mathcal{I}_{2}$,

$(c)\left\{\begin{array}{c}(u, s) \in \mathbb{N} \times \mathbb{N}: \frac{1}{h_{u s}} \sum_{(k, l) \in J_{u s}} \phi\left(\widehat{F} x_{k l}-\xi, t\right)>1-\varepsilon \\ \text { and } \frac{1}{h_{u s}} \sum_{(k, l) \in J_{u s}} \omega\left(\widehat{F} x_{k l}-\xi, t\right)<\varepsilon\end{array}\right\} \in \mathcal{F}\left(\mathcal{I}_{2}\right)$,

(d) $\left\{(u, s) \in \mathbb{N} \times \mathbb{N}: \frac{1}{h_{u s}} \sum_{(k, l) \in J_{u s}} \phi\left(\widehat{F} x_{k l}-\xi, t\right)>1-\varepsilon\right\} \in \mathcal{F}\left(\mathcal{I}_{2}\right)$ and

$\left\{(u, s) \in \mathbb{N} \times \mathbb{N}: \frac{1}{h_{u s}} \sum_{(k, l) \in J_{u s}} \omega\left(\widehat{F} x_{k l}-\xi, t\right)<\varepsilon\right\} \in \mathcal{F}\left(\mathcal{I}_{2}\right)$ and

(e) $F \mathcal{I}_{\theta_{u s}}^{(\phi, \omega)}-\lim \phi\left(\widehat{F} x_{k l}-\xi, t\right)=1$ and $F \mathcal{I}_{\theta_{u s}}^{(\phi, \omega)}-\lim \omega\left(\widehat{F} x_{k l}-\xi, t\right)=0$.

Theorem 2.2. If a sequence $x=\left(x_{k l}\right)$ in IFNS is Fibonacci lacunary $\mathcal{I}_{2}$-convergent with regards to the IFN $(\phi, \omega)$, then $F \mathcal{I}_{\theta_{u s}}^{(\phi, \omega)}-\lim x$ is unique.

Proof. Assume that $F \mathcal{I}_{\theta_{u s}}^{(\phi, \omega)}-\lim x=\xi_{1}$ and $F \mathcal{I}_{\theta_{u s}}^{(\phi, \omega)}-\lim x=\xi_{2}$. Given $\varepsilon \in(0,1)$, select $\gamma \in(0,1)$ such that $(1-\gamma) *(1-\gamma)>1-\varepsilon$ and $\gamma \diamond \gamma<\varepsilon$. Then, for any $t>0$, take the following sets:

$$
\begin{aligned}
& K_{\phi, 1}(\gamma, t)=\left\{(u, s) \in \mathbb{N} \times \mathbb{N}: \frac{1}{h_{u s}} \sum_{(k, l) \in J_{u s}} \phi\left(\widehat{F} x_{k l}-\xi_{1}, \frac{t}{2}\right) \leq 1-\gamma\right\}, \\
& K_{\phi, 2}(\gamma, t)=\left\{(u, s) \in \mathbb{N} \times \mathbb{N}: \frac{1}{h_{u s}} \sum_{(k, l) \in J_{u s}} \phi\left(\widehat{F} x_{k l}-\xi_{2}, \frac{t}{2}\right) \leq 1-\gamma\right\}, \\
& K_{\omega, 1}(\gamma, t)=\left\{(u, s) \in \mathbb{N} \times \mathbb{N}: \frac{1}{h_{u s}} \sum_{(k, l) \in J_{u s}} \omega\left(\widehat{F} x_{k l}-\xi_{1}, \frac{t}{2}\right) \geq \gamma\right\}, \\
& K_{\omega, 2}(\gamma, t)=\left\{(u, s) \in \mathbb{N} \times \mathbb{N}: \frac{1}{h_{u s}} \sum_{(k, l) \in J_{u s}} \omega\left(\widehat{F} x_{k l}-\xi_{2}, \frac{t}{2}\right) \geq \gamma\right\} .
\end{aligned}
$$

Since $F \mathcal{I}_{\theta_{u s}}^{(\phi, \omega)}-\lim x=\xi_{1}$, applying Lemma 2.1, we get $K_{\phi, 1}(\gamma, t) \in \mathcal{I}_{2}$ and $K_{\omega, 1}(\gamma, t) \in \mathcal{I}_{2}$ for every $t>0$. Using $F \mathcal{I}_{\theta_{u s}}^{(\phi, \omega)}-\lim x=\xi_{2}$, we have $K_{\phi, 2}(\gamma, t) \in \mathcal{I}_{2}$ and $K_{\omega, 2}(\gamma, t) \in \mathcal{I}_{2}$ for all $t>0$.

Now, take $K_{\phi, \omega}(\gamma, t)=\left(K_{\phi, 1}(\gamma, t) \cup K_{\phi, 2}(\gamma, t)\right) \cap\left(K_{\omega, 1}(\gamma, t) \cup K_{\omega, 2}(\gamma, t)\right)$. Then, $K_{\phi, \omega}(\gamma, t) \in \mathcal{I}_{2}$. This gives that $K_{\phi, \omega}^{c}(\gamma, t) \neq \emptyset$ in $\mathcal{F}\left(\mathcal{I}_{2}\right)$. If $(u, s) \in K_{\phi, \omega}^{c}(\gamma, t)$, first, contemplate the case $(u, s) \in\left(K_{\phi, 1}^{c}(\gamma, t) \cap K_{\phi, 2}^{c}(\gamma, t)\right)$. Then, we get

$$
\frac{1}{h_{u s}} \sum_{(k, l) \in J_{u s}} \phi\left(\widehat{F} x_{k l}-\xi_{1}, \frac{t}{2}\right)>1-\gamma \text { and } \frac{1}{h_{u s}} \sum_{(k, l) \in J_{u s}} \phi\left(\widehat{F} x_{k l}-\xi_{2}, \frac{t}{2}\right)>1-\gamma .
$$

Now, obviously, we will get a $(p, q) \in \mathbb{N} \times \mathbb{N}$ such that

$$
\phi\left(\widehat{F} x_{p q}-\xi_{1}, \frac{t}{2}\right)>\frac{1}{h_{u s}} \sum_{(k, l) \in J_{u s}} \phi\left(\widehat{F} x_{k l}-\xi_{1}, \frac{t}{2}\right)>1-\gamma
$$

and

$$
\phi\left(\widehat{F} x_{p q}-\xi_{2}, \frac{t}{2}\right)>\frac{1}{h_{u s}} \sum_{(k, l) \in J_{u s}} \phi\left(\widehat{F} x_{k l}-\xi_{2}, \frac{t}{2}\right)>1-\gamma
$$


(That is, consider $\max \left\{\phi\left(\widehat{F} x_{k l}-\xi_{1}, \frac{t}{2}\right), \phi\left(\widehat{F} x_{k l}-\xi_{2}, \frac{t}{2}\right):(k, l) \in J_{u s}\right\}$ and select that $(k, l)$ as $(p, q)$ for which the maximum occurs).

Then, we obtain

$$
\begin{aligned}
\phi\left(\xi_{1}-\xi_{2}, t\right) & \geq \phi\left(\widehat{F} x_{p q}-\xi_{1}, \frac{t}{2}\right) * \phi\left(\widehat{F} x_{p q}-\xi_{2}, \frac{t}{2}\right) \\
& >(1-\gamma) *(1-\gamma)>1-\varepsilon .
\end{aligned}
$$

Since $\varepsilon>0$ is arbitrary, we get $\phi\left(\xi_{1}-\xi_{2}, t\right)=1$ for each $t>0$, which gives that $\xi_{1}=\xi_{2}$. At the same time, if $(u, s) \in K_{\phi, 1}^{c}(\gamma, t) \cap K_{\phi, 2}^{c}(\gamma, t)$, then by using the similar method, it can be demonstrated that $\omega\left(\xi_{1}-\xi_{2}, t\right)<\varepsilon$, for arbitrary $\varepsilon>0$ and for every $t>0$, and so $\xi_{1}=\xi_{2}$. Hence, in all cases, we deduce that $F \mathcal{I}_{\theta_{u s}}^{(\phi, \omega)}-\lim x$ is unique.

Theorem 2.3. If $(\phi, \omega)^{\theta_{u s}}-\lim F x=\xi$, then $F \mathcal{I}_{\theta_{u s}}^{(\phi, \omega)}-\lim x=\xi$.

Proof. Let $(\phi, \omega)^{\theta_{u s}}-\lim F x=\xi$. Then, for every $t>0$ and $\varepsilon \in(0,1)$, there is $r_{0} \in \mathbb{N}$ such that

$$
\frac{1}{h_{u s}} \sum_{(k, l) \in J_{u s}} \phi\left(\widehat{F} x_{k l}-\xi, t\right)>1-\varepsilon \text { and } \frac{1}{h_{u s}} \sum_{(k, l) \in J_{u s}} \omega\left(\widehat{F} x_{k l}-\xi, t\right)<\varepsilon
$$

for all $u, s \geq r_{0}$. Therefore, we obtain

$$
\begin{aligned}
& A=\left\{\begin{array}{c}
(u, s) \in \mathbb{N} \times \mathbb{N}: \frac{1}{h_{u s}} \sum_{(k, l) \in J_{u s}} \phi\left(\widehat{F} x_{k l}-\xi, t\right) \leq 1-\varepsilon \\
\text { or } \frac{1}{h_{u s}} \sum_{(k, l) \in J_{u s}} \omega\left(\widehat{F} x_{k l}-\xi, t\right) \geq \varepsilon
\end{array}\right\} \\
& \subseteq\left\{(1,1),(2,2), \ldots,\left(k_{0}-1, k_{0}-1\right)\right\} .
\end{aligned}
$$

But, with $\mathcal{I}_{2}$ being admissible ideal, we get $A \in \mathcal{I}_{2}$. Hence, $F \mathcal{I}_{\theta_{u s}}^{(\phi, \omega)}-\lim x=\xi$.

Theorem 2.4. If $(\phi, \omega)^{\theta_{u s}}-\lim F x=\xi$, then there exists a subsequence $\left(x_{k^{\prime}(u) l^{\prime}(s)}\right)$ of $x$ such that $(\phi, \omega)^{\theta_{u s}}-\lim F x_{k^{\prime}(u) l^{\prime}(s)}=$ $\xi$.

Proof. Let $(\phi, \omega)^{\theta_{u s}}-\lim F x=\xi$. Then, for every $t>0$ and $\varepsilon \in(0,1)$, there exists $r_{0} \in \mathbb{N}$ such that

$$
\frac{1}{h_{u s}} \sum_{(k, l) \in J_{u s}} \phi\left(\widehat{F} x_{k l}-\xi, t\right)>1-\varepsilon \text { and } \frac{1}{h_{u s}} \sum_{(k, l) \in J_{u s}} \omega\left(\widehat{F} x_{k l}-\xi, t\right)<\varepsilon
$$

for all $u, s \geq r_{0}$. Obviously, for each $u, s \geq r_{0}$, we can select $\left(k^{\prime}(u), l^{\prime}(s)\right) \in J_{u s}$ such that

$$
\begin{gathered}
\phi\left(\widehat{F} x_{k^{\prime}(u) l^{\prime}(s)}-\xi, t\right)>\frac{1}{h_{u s}} \sum_{(k, l) \in J_{u s}} \phi\left(\widehat{F} x_{k l}-\xi, t\right)>1-\varepsilon \\
\text { and } \omega\left(\widehat{F} x_{k^{\prime}(u) l^{\prime}(s)}-\xi, t\right)<\frac{1}{h_{u s}} \sum_{(k, l) \in J_{u s}} \omega\left(\widehat{F} x_{k l}-\xi, t\right)<\varepsilon .
\end{gathered}
$$

It follows that $(\phi, \omega)^{\theta_{u s}}-\lim F x_{k^{\prime}(u) l^{\prime}(s)}=\xi$.

Definition 2.3. A double sequence $x=\left(x_{j k}\right)$ in IFNS is named as Fibonacci lacunary Cauchy with regards to the $\operatorname{IFN}(\phi, \omega)$ if, for each $\varepsilon>0$ and $t>0$, there exist $N=N(\varepsilon)$ and $M=M(\varepsilon)$ such that, for all $j, p \geq N, k, q \geq M$,

$$
\frac{1}{h_{u s}} \sum_{(j, k),(p, q) \in J_{u s}} \phi\left(\widehat{F} x_{j k}-\widehat{F} x_{p q}, t\right)>1-\varepsilon \text { and } \frac{1}{h_{u s}} \sum_{(j, k),(p, q) \in J_{u s}} \omega\left(\widehat{F} x_{j k}-\widehat{F} x_{p q}, t\right)<\varepsilon .
$$

Definition 2.4. A double sequence $x=\left(x_{j k}\right)$ in IFNS is named as Fibonacci lacunary $\mathcal{I}_{2}$-Cauchy with regards to the $\operatorname{IFN}(\phi, \omega)$ if, for every $\varepsilon \in(0,1)$ and $t>0$, there exists $(p, q) \in \mathbb{N} \times \mathbb{N}$ fulfilling

$$
\left\{\begin{array}{c}
(u, s) \in \mathbb{N} \times \mathbb{N}: \frac{1}{h_{u s}} \sum_{(j, k) \in J_{u s}} \phi\left(\widehat{F} x_{j k}-\widehat{F} x_{p q}, t\right)>1-\varepsilon \\
\text { and } \frac{1}{h_{u s}} \sum_{(j, k) \in J_{u s}} \omega\left(\widehat{F} x_{j k}-\widehat{F} x_{p q}, t\right)<\varepsilon
\end{array}\right\} \in \mathcal{F}\left(\mathcal{I}_{2}\right) .
$$


Definition 2.5. A double sequence $x=\left(x_{j k}\right)$ in IFNS is named as Fibonacci lacunary $\mathcal{I}_{2}^{*}$-Cauchy with regards to the IFN $(\phi, \omega)$ if there is a subset $M=\left\{\left(j_{m}, k_{m}\right): j_{1}<j_{2}<\ldots ; k_{1}<k_{2}<\ldots\right\}$ of $\mathbb{N} \times \mathbb{N}$ such that the set $M^{\prime}=$ $\left\{(u, s) \in \mathbb{N} \times \mathbb{N}:\left(j_{u}, k_{s}\right) \in J_{u s}\right\} \in \mathcal{F}\left(\mathcal{I}_{2}\right)$ and the subsequence $\left(x_{j_{u} k_{s}}\right)$ is a Fibonacci lacunary Cauchy sequence with regards to the IFN $(\phi, \omega)$.

Theorem 2.5. A double sequence $x=\left(x_{j k}\right)$ is Fibonacci lacunary $\mathcal{I}_{2}$-convergent with regards to the IFN $(\phi, \omega)$ iff it is Fibonacci lacunary $\mathcal{I}_{2}$-Cauchy with regards to $(\phi, \omega)$.

Proof. Let $x=\left(x_{j k}\right)$ be Fibonacci lacunary $\mathcal{I}_{2}$-convergent to $\xi$ with regards to the $\operatorname{IFN}(\phi, \omega)$. Then

$$
\left\{\begin{array}{c}
(u, s) \in \mathbb{N} \times \mathbb{N}: \frac{1}{h_{u s}} \sum_{(j, k) \in J_{u s}} \phi\left(\widehat{F} x_{j k}-\xi, \frac{t}{2}\right) \leq 1-\varepsilon \\
\text { or } \frac{1}{h_{u s}} \sum_{(j, k) \in J_{u s}} \omega\left(\widehat{F} x_{j k}-\xi, \frac{t}{2}\right) \geq \varepsilon
\end{array}\right\} \in \mathcal{I}_{2} .
$$

Specifically, for $j=M, k=N$

$$
\left\{\begin{array}{c}
(u, s) \in \mathbb{N} \times \mathbb{N}: \frac{1}{h_{u s}} \sum_{(M, N) \in J_{u s}} \phi\left(\widehat{F} x_{M N}-\xi, \frac{t}{2}\right) \leq 1-\varepsilon \\
\text { or } \frac{1}{h_{u s}} \sum_{(M, N) \in J_{u s}} \omega\left(\widehat{F} x_{M N}-\xi, \frac{t}{2}\right) \geq \varepsilon
\end{array}\right\} \in \mathcal{I}_{2} .
$$

Since

$$
\begin{aligned}
\phi\left(\widehat{F} x_{j k}-\widehat{F} x_{M N}, t\right) & =\phi\left(\widehat{F} x_{j k}-\xi-\widehat{F} x_{M N}+\xi, \frac{t}{2}+\frac{t}{2}\right) \\
& \geq \phi\left(\widehat{F} x_{j k}-\xi, \frac{t}{2}\right) * \phi\left(\widehat{F} x_{M N}-\xi, \frac{t}{2}\right)
\end{aligned}
$$

and

$$
\omega\left(\widehat{F} x_{j k}-\widehat{F} x_{M N}, t\right) \leq \omega\left(x_{j k}-\xi, \frac{t}{2}\right) \diamond \omega\left(x_{M N}-\xi, \frac{t}{2}\right)
$$

we obtain

$$
\left\{\begin{array}{c}
(u, s) \in \mathbb{N} \times \mathbb{N}: \frac{1}{h_{u s}} \sum_{(j, k) \in J_{u s}} \mu\left(\widehat{F} x_{j k}-\widehat{F} x_{M N}, t\right) \leq 1-\varepsilon \\
\text { or } \frac{1}{h_{u s}} \sum_{(j, k) \in J_{u s}} \omega\left(\widehat{F} x_{j k}-\widehat{F} x_{M N}, t\right) \geq \varepsilon
\end{array}\right\} \in \mathcal{I}_{2} .
$$

That is, $x$ is Fibonacci $\mathcal{I}_{2}$-lacunary Cauchy with regards to $(\phi, \omega)$.

In contrast, let $x=\left(x_{j k}\right)$ be Fibonacci $\mathcal{I}_{2}$-lacunary Cauchy but not Fibonacci lacunary $\mathcal{I}_{2}$-convergent with regards to the IFN $(\phi, \omega)$. Then, there are $N$ and $M$ such that the set $A(\varepsilon, t) \in \mathcal{I}_{2}$, where

$$
A(\varepsilon, t)=\left\{\begin{array}{c}
(u, s) \in \mathbb{N} \times \mathbb{N}: \frac{1}{h_{u s}} \sum_{(j, k) \in J_{u s}} \phi\left(x_{j k}-x_{M N}, t\right) \leq 1-\varepsilon \\
\text { or } \frac{1}{h_{u s}} \sum_{(j, k) \in J_{u s}} \omega\left(x_{k l}-x_{M N}, t\right) \geq \varepsilon
\end{array}\right\}
$$

and also $B(\varepsilon, t) \in \mathcal{I}_{2}$, where

$$
B(\varepsilon, t)=\left\{\begin{array}{c}
(u, s) \in \mathbb{N} \times \mathbb{N}: \frac{1}{h_{u s}} \sum_{(j, k) \in J_{u s}} \phi\left(\widehat{F} x_{j k}-\xi, \frac{t}{2}\right) \leq 1-\varepsilon \\
\text { or } \frac{1}{h_{u s}} \sum_{(j, k) \in J_{u s}} \omega\left(\widehat{F} x_{j k}-\xi, \frac{t}{2}\right) \geq \varepsilon
\end{array}\right\} .
$$

Since

$$
\phi\left(\widehat{F} x_{j k}-\widehat{F} x_{M N}, t\right) \geq 2 \phi\left(\widehat{F} x_{j k}-\xi, \frac{t}{2}\right)>1-\varepsilon,
$$


and

$$
\omega\left(\widehat{F} x_{j k}-\widehat{F} x_{M N}, t\right) \leq 2 \omega\left(\widehat{F} x_{j k}-\xi, \frac{t}{2}\right)<\varepsilon,
$$

if $\phi\left(\widehat{F} x_{j k}-\xi, \frac{t}{2}\right)>\frac{(1-\varepsilon)}{2}$ and $\omega\left(\widehat{F} x_{j k}-\xi, \frac{t}{2}\right)<\frac{\varepsilon}{2}$. Therefore,

$$
\left\{\begin{array}{c}
(u, s) \in \mathbb{N} \times \mathbb{N}: \frac{1}{h_{u s}} \sum_{(j, k) \in J_{u s}} \phi\left(\widehat{F} x_{j k}-\widehat{F} x_{M N}, t\right)>1-\varepsilon \\
\text { or } \frac{1}{h_{u s}} \sum_{(j, k) \in J_{u s}} \omega\left(\widehat{F} x_{j k}-\widehat{F} x_{M N}, t\right)<\varepsilon
\end{array}\right\} \in \mathcal{I}_{2},
$$

that is, $A^{c}(\varepsilon, t) \in \mathcal{I}_{2}$ and hence, $A(\varepsilon, t) \in \mathcal{F}\left(\mathcal{I}_{2}\right)$, which leads to a contradiction. Hence $x$ must be Fibonacci lacunary $\mathcal{I}_{2}$-convergent with regards to the $\operatorname{IFN}(\phi, \omega)$.

Theorem 2.6. If $\left(\rho_{u s}\right)$ is a double lacunary refinement of $\theta_{u s}$ and $F \mathcal{I}_{\rho_{u s}}^{(\phi, \omega)}-\lim x=\xi$, then $F \mathcal{I}_{\theta_{u s}}^{(\phi, \omega)}-\lim x=\xi$.

Proof. Presume that each $\mathcal{I}_{u s}$ of $\theta_{u s}$ involves the points $\left(\bar{k}_{u, i}, \bar{l}_{s, j}\right)_{i, j=1}^{v(u), w(s)}$ of $\left(\rho_{u s}\right)$ so that

$$
\begin{gathered}
k_{u-1}<\bar{k}_{u, 1}<\bar{k}_{u, 2}<\ldots<\bar{k}_{u, v(u)}=k_{u}, \text { where } \bar{l}_{u, i}=\left(\bar{k}_{u, i-1}, \bar{k}_{u, i}\right], \\
l_{s-1}<\bar{l}_{s, 1}<\bar{l}_{s, 2}<\ldots<\bar{l}_{s, w(s)}=l_{s}, \text { where } \bar{J}_{s, j}=\left(\bar{l}_{s, j-1}, \bar{l}_{s, j}\right],
\end{gathered}
$$

and

$$
\bar{J}_{u, s, i, j}=\left\{(k, l): \bar{k}_{u, i-1}<k \leq \bar{k}_{u} ; \bar{l}_{s, j-1}<l \leq \bar{l}_{s}\right\}
$$

for all $u, s$ and $v(u) \geq 1, w(s) \geq 1$ this gives that $\left(k_{u}, l_{s}\right) \subseteq\left(\bar{k}_{u}, \bar{l}_{s}\right)$. Let $\left(\bar{J}_{i j}\right)_{i, j=1,1}^{\infty, \infty}$ be the sequence of abutting blocks of $\left(\bar{J}_{u, s, i, j}\right)$ ordered by increasing a lower right index points. Since $F \mathcal{I}_{\rho_{u s}}^{(\phi, \omega)}-\lim x=\xi$, we obtain the following for each $t>0$ and $\varepsilon \in(0,1)$

$$
\left\{\begin{array}{c}
(i, j) \in \mathbb{N} \times \mathbb{N}: \frac{1}{\bar{h}_{i j}} \sum_{\bar{J}_{i j} \subset J_{u s}} \phi\left(\widehat{F} x_{k l}-\xi, t\right) \leq 1-\varepsilon \\
\text { or } \frac{1}{\bar{h}_{i j}} \sum_{\bar{J}_{i j} \subset J_{u s}} \omega\left(\widehat{F} x_{k l}-\xi, t\right) \geq \varepsilon
\end{array}\right\} \in \mathcal{I}_{2} .
$$

As before, we take $h_{u s}=h_{u} \bar{h}_{s} ; \bar{h}_{u i}=\bar{k}_{u i}-\bar{k}_{u, i-1} ; \bar{h}_{s j}=\bar{l}_{s, j}-\bar{l}_{s, j-1}$.

For each $t>0$ and $\varepsilon \in(0,1)$ we get

$$
\begin{gathered}
\left\{\begin{array}{r}
(u, s) \in \mathbb{N} \times \mathbb{N}: \frac{1}{h_{u s}} \sum_{(k, l) \in J_{u s}} \phi\left(\widehat{F} x_{k l}-\xi, t\right) \leq 1-\varepsilon \\
\text { or } \frac{1}{h_{u s}} \sum_{(k, l) \in J_{u s}} \omega\left(\widehat{F} x_{k l}-\xi, t\right) \geq \varepsilon
\end{array}\right\} \\
\subseteq\left\{\begin{array}{c}
(u, s) \in \mathbb{N} \times \mathbb{N}: \frac{1}{h_{u s}} \sum_{(k, l) \in J_{u s}}\{(i, j) \in \mathbb{N} \times \mathbb{N}: \\
\frac{1}{\bar{h}_{i j}} \sum_{\bar{J}_{i j} \subset J_{u s} ;(k, l) \in \bar{J}_{i j}} \phi\left(\widehat{F} x_{k l}-\xi, t\right) \leq 1-\varepsilon \\
\operatorname{or}_{\frac{1}{\bar{h}_{i j}}} \sum_{\bar{J}_{i j} \subset J_{u s} ;(k, l) \in \bar{J}_{i j}} \omega\left(\widehat{F} x_{k l}-\xi, t\right) \geq \varepsilon
\end{array}\right\} .
\end{gathered}
$$

By (2.1), for each $t>0$ and $\varepsilon \in(0,1)$ if we define

$$
t_{i j}=\left(\begin{array}{c}
\frac{1}{\bar{h}_{i j}} \sum_{(k, l) \in \bar{J}_{i j}} \phi\left(\widehat{F} x_{k l}-\xi, t\right) \leq 1-\varepsilon \\
\text { or } \frac{1}{\bar{h}_{i, j}} \sum_{\bar{J}_{i j} \subset J_{u s} ;(k, l) \in \bar{J}_{i j}} \omega\left(\widehat{F} x_{k l}-\xi, t\right) \geq \varepsilon
\end{array}\right)_{i, j=1}^{\infty, \infty},
$$


then $\left(t_{i, j}\right)$ is a Pringsheim null sequence. The transformation

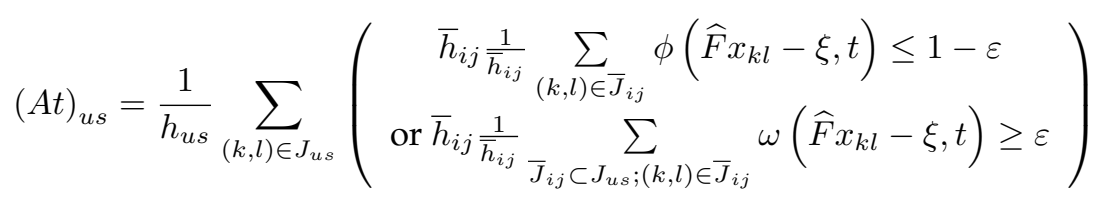

fulfills all situations for a matrix transformation to map a Pringsheim null sequence into a Pringsheim null sequence. Hence, $F \mathcal{I}_{\theta_{u s}}^{(\phi, \omega)}-\lim x=\xi$.

Definition 2.6. Let $(X, \phi, \omega, *, \diamond)$ be an IFNS.

(a) An element $\xi \in X$ is named as Fibonacci lacunary $\mathcal{I}_{2}$-limit point of $x=\left(x_{k l}\right)$ if there is set $M=\left\{\left(k_{1}, l_{1}\right)<\left(k_{2}, l_{2}\right)<\ldots<\left(k_{u}, l_{s}\right)<\ldots\right\} \subset \mathbb{N} \times \mathbb{N}$ such that the set

$$
M^{\prime}=\left\{(u, s) \in \mathbb{N} \times \mathbb{N}:\left(k_{u}, l_{s}\right) \in J_{u s}\right\} \notin \mathcal{I}_{2}
$$

and $(\phi, \omega)^{\theta_{u s}}-\lim F x_{k_{u} l_{s}}=\xi$. $\varepsilon \in(0,1)$, we get

(b) $\xi \in X$ is named as Fibonacci lacunary $\mathcal{I}_{2}$-cluster point of $x=\left(x_{k l}\right)$ if, for every $t>0$ and

$$
\left\{\begin{array}{c}
(u, s) \in \mathbb{N} \times \mathbb{N}: \frac{1}{h_{u s}} \sum_{(k, l) \in J_{u s}} \phi\left(\widehat{F} x_{k l}-\xi, t\right)>1-\varepsilon \\
\text { and } \frac{1}{h_{u s}} \sum_{(k, l) \in J_{u s}} \omega\left(\widehat{F} x_{k l}-\xi, t\right)<\varepsilon
\end{array}\right\} \notin \mathcal{I}_{2} .
$$

$\Lambda_{(\phi, \omega)^{\theta} u s}^{F \mathcal{I}_{2}}(x)$ and $\Gamma_{(\phi, \omega)^{\theta} u s}^{F \mathcal{I}_{2}}(x)$ indicate the set of all Fibonacci lacunary $\mathcal{I}_{2}$-limit points and the set of all Fibonacci lacunary $\mathcal{I}_{2}$-cluster points in IFNS, respectively.

Theorem 2.7. For every sequence $x=\left(x_{k l}\right)$ in IFNS, we have $\Lambda_{(\phi, \omega)^{\theta_{u s}}}^{F \mathcal{I}_{2}}(x) \subseteq \Gamma_{(\phi, \omega)^{\theta_{u s}}}^{F \mathcal{I}_{2}}(x)$.

Proof. Let $\xi \in \Lambda_{(\phi, \omega)^{\theta} u s}^{F \mathcal{I}_{2}}(x)$. Then, there is a set $M \subset \mathbb{N} \times \mathbb{N}$ such that the set $M^{\prime} \notin \mathcal{I}_{2}$, where $M$ and $M^{\prime}$ are as in Definition 2.6, fulfills $(\phi, \omega)^{\theta_{u s}}-\lim F x_{k_{u} l_{s}}=\xi$. Hence, for every $t>0$ and $\varepsilon \in(0,1)$, there are $u_{0}, s_{0} \in \mathbb{N}$ such that

$$
\frac{1}{h_{u s}} \sum_{(k, l) \in J_{u s}} \phi\left(\widehat{F} x_{k_{u} l_{s}}-\xi, t\right)>1-\varepsilon \text { and } \frac{1}{h_{u s}} \sum_{(k, l) \in J_{u s}} \omega\left(\widehat{F} x_{k_{u} l_{s}}-\xi, t\right)<\varepsilon
$$

for all $u \geq u_{0}, s \geq s_{0}$. Therefore,

$$
\begin{aligned}
B= & \left\{\begin{array}{c}
(u, s) \in \mathbb{N} \times \mathbb{N}: \frac{1}{h_{u s}} \sum_{(k, l) \in J_{u s}} \phi\left(\widehat{F} x_{k l}-\xi, t\right)>1-\varepsilon \\
\text { and } \frac{1}{h_{u s}} \sum_{(k, l) \in J_{u s}} \omega\left(\widehat{F} x_{k l}-\xi, t\right)<\varepsilon
\end{array}\right\} \\
& \supseteq M^{\prime} \backslash\left\{\left(k_{1}, l_{1}\right)<\left(k_{2}, l_{2}\right)<\ldots<\left(k_{u_{0}}, l_{s_{0}}\right)\right\} .
\end{aligned}
$$

Now, with $\mathcal{I}_{2}$ being admissible, we must have $M^{\prime} \backslash\left\{\left(k_{1}, l_{1}\right)<\left(k_{2}, l_{2}\right)<\ldots<\left(k_{u_{0}}, l_{s_{0}}\right)\right\} \notin \mathcal{I}_{2}$ and as such $B \notin \mathcal{I}_{2}$. Hence, $\xi \in \Gamma_{(\phi, \omega)^{\theta} \text { us }}^{F \mathcal{I}_{2}}(x)$.

Theorem 2.8. The following observations are equivalent.

(a) $\xi$ is Fibonacci lacunary $\mathcal{I}_{2}$-limit point of $x$. and

(b) There are two sequences $y=\left(y_{k l}\right)$ and $z=\left(z_{k l}\right)$ in IFNS such that $x=y+z$ and $(\phi, \omega)^{\theta_{u s}}-\lim F y=\xi$

$$
\left\{(u, s) \in \mathbb{N} \times \mathbb{N}:(k, l) \in J_{u s}, z_{k l} \neq \overline{0}\right\} \in \mathcal{I}_{2} .
$$


Proof. Presume that $(a)$ holds. Then there are $M$ and $M^{\prime}$ are as in Definition 2.6 such that $M^{\prime} \notin \mathcal{I}_{2}$ and $(\phi, \omega)^{\theta_{u s}}-$ $\lim F x=\xi$. Take the sequences $y$ and $z$ as follows:

$$
y_{k l}= \begin{cases}x_{k l}, & \text { if }(k, l) \in J_{u s},(u, s) \in M^{\prime} \\ \xi, & \text { otherwise }\end{cases}
$$

and

$$
z_{k l}= \begin{cases}\overline{0}, & \text { if }(k, l) \in J_{u s},(u, s) \in M^{\prime} \\ x_{k l}-\xi, & \text { otherwise. }\end{cases}
$$

It is adequate to think the case $(k, l) \in J_{u s}$ such that $(u, s) \in \mathbb{N} \times \mathbb{N} \backslash M^{\prime}$. Then, for each $t>0$ and $\varepsilon \in(0,1)$. Then, we have $\phi\left(\widehat{F} y_{k l}-\xi, t\right)=1>1-\varepsilon$ and $\omega\left(\widehat{F} y_{k l}-\xi, t\right)=0<\varepsilon$. Thus, we write

$$
\frac{1}{h_{u s}} \sum_{(k, l) \in J_{u s}} \phi\left(\widehat{F} y_{k l}-\xi, t\right)=1>1-\varepsilon \text { and } \frac{1}{h_{u s}} \sum_{(k, l) \in J_{u s}} \omega\left(\widehat{F} y_{k l}-\xi, t\right)=0<\varepsilon
$$

Hence, $(\phi, \omega)^{\theta_{u s}}-\lim y=\xi$. Now, we have

$$
\left\{(u, s) \in \mathbb{N} \times \mathbb{N}:(k, l) \in J_{u s}, z_{k l} \neq \overline{0}\right\} \subset \mathbb{N} \times \mathbb{N} \backslash M^{\prime} .
$$

But $\mathbb{N} \times \mathbb{N} \backslash M^{\prime} \in \mathcal{I}_{2}$, and so

$$
\left\{(u, s) \in \mathbb{N} \times \mathbb{N}:(k, l) \in J_{u s}, z_{k l} \neq \overline{0}\right\} \in \mathcal{I}_{2} .
$$

Now, presume that $(b)$ holds. Let $M^{\prime}=\left\{(u, s) \in \mathbb{N} \times \mathbb{N}:(k, l) \in J_{u s}, z_{k l}=\overline{0}\right\}$. Then, obviously $M^{\prime} \in \mathcal{F}\left(\mathcal{I}_{2}\right)$ and so it is an infinite set. Construct the set

$$
M=\left\{\left(k_{1}, l_{1}\right)<\left(k_{2}, l_{2}\right)<\ldots<\left(k_{u}, l_{s}\right)<\ldots\right\} \subset \mathbb{N} \times \mathbb{N}
$$

such that $\left(k_{u}, l_{s}\right) \in J_{u s}$ and $z_{k_{u} l_{s}}=\overline{0}$. Since $x_{k_{u} l_{s}}=y_{k_{u} l_{s}}$ and $(\phi, \omega)^{\theta_{u s}}-\lim F y=\xi$ we obtain $(\phi, \omega)^{\theta_{u s}}-$ $\lim F x_{k_{u} l_{s}}=\xi$.

\section{Acknowledgment.}

We thank the reviewer for their insightful comments and suggestions that helped us improve the paper.

\section{Funding}

There is no funding for this work.

\section{Availability of data and materials}

Not applicable.

\section{Competing interests}

The authors declare that they have no competing interests.

\section{Author's contributions}

All authors contributed equally to the writing of this paper. All authors read and approved the final manuscript.

\section{References}

[1] Atanassov, K.T.: Intuitionistic fuzzy sets. Fuzzy Sets and Syst. 20, 87-96 (1986).

[2] Debnath, P.: Lacunary ideal convergence in intuitionistic fuzzy normed linear spaces. Comput. Math. Appl. 63, 708-715 (2012). 
[3] Dündar, E., Ulusu, U., Pancaroğlu, N.: Strongly $\mathcal{I}_{2}$-lacunary convergence and $\mathcal{I}_{2}$-lacunary Cauchy double sequences of sets. Aligarh Bull. Math. 35 (1-2), 1-15 (2016).

[4] Dündar, E., Ulusu, U., Aydın, B.: $\mathcal{I}_{2}$-lacunary statistical convergence of double sequences of sets. Konuralp J. Math. 5 (1), 1-10 (2017).

[5] Fast, H.: (1951). Sur la convergence statistique. Colloq. Math. 2, 241-244 (1951).

[6] Fridy, J.A., Orhan, C.: Lacunary statistical convergence. Pacific J. Math. 160 (1), 43-51 (1993).

[7] Goonatilake S.: Toward a Global Science. Indiana University Press. (1998).

[8] Hazarika, B.: Lacunary ideal convergence of multiple sequences. J. Egyptian Math. Soc. 24, 54-59 (2016).

[9] Kara, E.E., Başarı, M.: An application of Fibonacci numbers into infinite Toeplitz matrices. Casp. J. Math. Sci. 1 (1), 43-47 (2012).

[10] Kara, E.E.: Some topological and geometrical properties of new Banach sequence spaces. J. Inequal. Appl. 38 (2013), (2013).

[11] Karakuş, S., Demirci, K., Duman, O.: Statistical convergence on intuitionistic fuzzy normed spaces. Chaos Solitons Fractals. 35, 763-769 (2008).

[12] Kirişçi, M., Karaisa, A.: Fibonacci statistical convergence and Korovkin type approximation theorems. J. Inequal. Appl. 2017 (229), 1-15 (2017).

[13] Kişi, Ö., Tuzcuoğlu, İ.: Fibonacci lacunary statistical convergence in intuitionistic fuzzy normed linear spaces. J. Progress Res. Soc. Sci. 16 (3), 3001-3007 (2020).

[14] Kişi, Ö., Güler, E.: On Fibonacci ideal convergence of double sequences in intuitionistic fuzzy normed linear spaces. Turk. J. Math. Comput. Sci. 11, 46-55 (2019).

[15] Koshy, T.: Fibonacci and Lucas Numbers with Applications. Wiley, New York. (2001).

[16] Kostyrko, P., Salat, T., Wilczynsski, W.: I-convergence. Real Anal. Exchange. 6 (2), 669-686 (2001).

[17] Mursaleen, M., Edely, O.H.: Statistical convergence of double sequences. J. Math. Anal. Appl. 288, 223-231 (2003).

[18] Mursaleen, M., Mohiuddine, S.A.: Statistical convergence of double sequences in intuitionistic fuzzy normed space. Chaos Solitons Fractals. 41, 2414-2421 (2009).

[19] Mursaleen, M., Mohiuddine, S.A., Edely, O.H.H.: On the ideal convergence of double sequences in intuitionistic fuzzy normed spaces. Comput. Math. Appl. 59, 603-611 (2010).

[20] Mursaleen, M., Mohiuddine, S.A.: On lacunary statistical convergence with respect to the intuitionistic fuzzy normed space. J. Comput. Appl. Math. 233 (2), 142-149 (2009).

[21] Nuray, F., Ulusu, U., Dündar, E.: Lacunary statistical convergence of double sequences of sets. Soft Comput. 20, 2883-2888 (2016).

[22] Park, J.H.: Intuitionistic fuzzy metric spaces. Chaos Solitons Fractals. 22, 1039-1046 (2004).

[23] Lael, F., Nourouzi, K.: Some results on the IF-normed spaces. Chaos Solitons Fractals. 37, 931-939 (2008).

[24] Savaş, E., Patterson, R.F.: Lacunary statistical convergence of double sequences. Math. Commun. 10, 55-61 (2005).

[25] Schweizer, B., Sklar, A.: Statistical metric spaces. Pacific J. Math. 10, 314-334. (1960).

[26] Temizer Ersoy, M., Furkan, H.: Distinguished supspaces in topological sequence spaces theory. AIMS Math. 5 (4), 2858-2868 (2020).

[27] Temizer Ersoy, M.: Some abelian, tauberian and core theorems related to the $(V, \lambda)$ summability. Univers. J. Math. Appl. 4 (2), 70-75. 
[28] Tripathy, B.C., Hazarika, B., Choudhary, B.B.: Lacunary I-convergent sequences. Kyungpook Math. J. 2 (4), 473-482 (2012).

[29] Tripathy, B.K., Tripathy, B.C.: On I-convergent double sequences. Soochow J. Math. 31 (4), 549-560 (2005).

[30] Zadeh, L.A.: Fuzzy sets. Inf.Control. 8, 338-353 (1965). (2021).

\section{Affiliations}

ÖMER KIŞI

AdDRESS: Bartın University, Dept. of Mathematics, 74100, Bartın-Turkey. E-MAIL: okisi@bartin.edu.tr

ORCID ID:0000-0001-6844-3092 